Highly Energetic Physical Processes and

Mechanisms for Emission from Astrophysical Plasmas

IAU Symposium, Vol. 195, 2000

P. C. H. Martens, S. Tsuruta, and M. A. Weber, eds.

\title{
Electron Acceleration by Strong DC Electric Fields in Extragalactic Jets
}

\author{
Y. E. Litvinenko \\ Institute for the Study of Earth, Oceans, and Space, University of New \\ Hampshire, Durham, NH 03824-3525, U.S.A.
}

\begin{abstract}
Fast magnetic reconnection in extragalactic jets leads to electron acceleration by the $\mathrm{DC}$ electric field in the reconnecting current sheet. The maximum electron energy $\left(\gamma>10^{6}\right)$ and the acceleration time $\left(<10^{6} \mathrm{~s}\right)$ are determined by the magnetic field dynamics in the sheet.
\end{abstract}

Many extragalactic jets originating in active galactic nuclei are sources of radiation that results from synchrotron emission by relativistic electrons. In some cases, continuous radiation extends to optical and even to X-ray frequencies, implying the presence of electrons with TeV energies (Eilek \& Hughes 1991). This paper demonstrates that the electric field associated with magnetic reconnection can accelerate these electrons locally all along the jet.

The electron energy gain in the reconnecting current sheet is determined by the potential drop experienced by a particle: $m_{\mathrm{e}} c^{2} \gamma=e E l_{\text {acc }}$. Here, $\gamma \gg 1$ is the electron Lorentz factor, $m_{\mathrm{e}}$ and $e$ are the electron mass and charge, $E$ is the electric field in the sheet, $l_{\text {acc }}$ is the acceleration length defined as the particle displacement along $\mathbf{E}$ in the sheet, and $c$ is the speed of light. Charged particle orbits in the sheet are determined by the magnetic field inside the sheet: $\mathbf{B}=-(y / a) B_{0} \hat{\mathbf{x}}+B_{\perp}(x) \hat{\mathbf{y}}+B_{\|} \hat{\mathbf{z}}$. Here, the field is approximated by the first nonzero terms in the Taylor expansion around $y=0$. The reconnection electric field $\mathbf{E}=E \hat{\mathbf{z}}$ is in the $z$-direction, and $a$ is the current sheet half-thickness. Both $B_{0}$ and the nonreconnecting component $B_{\|}$may be assumed constant.

A sufficiently strong longitudinal field $B_{\|}$magnetizes the electrons and makes them follow the field lines (Litvinenko 1996). Acceleration will cease when the particles leave the sheet. Integrating the magnetic field-line equations gives the acceleration length $l_{\text {acc }}=a B_{\|} / B_{\perp}$. This leads to the electron energy gain:

$$
m_{\mathrm{e}} c^{2} \gamma=e E a B_{\|} / B_{\perp}
$$

Obviously, $l_{\text {acc }}$ and $\gamma$ are very large if $B_{\perp} \rightarrow 0$. The magnetic field projection onto the $x y$-plane has the geometry of a standard magnetic X-point at $x=y=0$ where $B_{\perp}=0$. This is where the field lines are "cut and reconnected". The spatial variation of the field is related to its temporal evolution. The reconnecting field lines move into the sheet with the speed $v_{\text {in }}$ and out of the sheet with the Alfvén speed $v_{\mathrm{A}}=B_{0} /\left(4 \pi m_{\mathrm{p}} n\right)^{1 / 2}$, and carry the magnetized particles with them. The reconnected field lines straighten out so that $B_{\perp}$ increases from zero at $x=0$ to a maximum value at the edges of the sheet $x= \pm w / 2$ for each reconnected field line, leading to a dependence $B_{\perp}=B_{\perp}(x)$ in a steady state. I 
assume for simplicity a constant speed of the reconnected field lines:

$$
B_{\perp}(t)=4\left\langle B_{\perp}\right\rangle v_{\mathrm{A}} t / w .
$$

The following numerical values are used. The reconnecting field component $B_{0}=10^{-4} \mathrm{G}$ and the particle density $n=10^{-4} \mathrm{~cm}^{-3}$ follow from numerous observations. The width $w$ and length $l_{\mathrm{cs}}$ of the sheet are of the order of the jet size $0.5 \mathrm{kpc}=1.5 \cdot 10^{21} \mathrm{~cm}$. The reconnecting and nonreconnecting components of the field are assumed to be the same: $B_{\|}=B_{0}$. Models for fast collisionless reconnection suggest that $\left\langle B_{\perp}\right\rangle / B_{0} \approx v_{\text {in }} / v_{\mathrm{A}} \approx 0.1$ (Hill 1975). The current sheet half-thickness $a$ is of the order of the ion skin depth $\left(m_{\mathrm{p}} c^{2} / 4 \pi n e^{2}\right)^{1 / 2}=$ $2 \cdot 10^{9} \mathrm{~cm}$. The electric field $E \approx 0.8 \cdot 10^{-6}$ statvolt $\mathrm{cm}^{-1}$ corresponds to the electric drift inflow speed $v_{\text {in }}=0.1 v_{\mathrm{A}} \approx 2.4 \cdot 10^{8} \mathrm{~cm} \mathrm{~s}^{-1}$.

The maximum electron energy can be estimated by noting that the magnetized electrons move almost along $\mathbf{B}$ inside the sheet, so their relativistic Lorentz factor increases with time as

$$
\gamma(t)=e E t / m_{\mathrm{e}} c .
$$

The maximum energy is determined by Eq. (1) with the time-dependent $B_{\perp}$ for a reconnected field line given by Eq. (2) and the time-dependent Lorentz factor given by Eq. (3). Making the substitutions leads to an equation for the maximum acceleration time, which is solved to give

$$
t_{\mathrm{acc}}=\frac{1}{2}\left(\frac{B_{\|}}{\left\langle B_{\perp}\right\rangle} \frac{w a}{c v_{\mathrm{A}}}\right)^{1 / 2} \approx 3.5 \cdot 10^{5} \mathrm{~s} .
$$

Substituting this into Eq. (3) gives the sought-after maximum electron Lorentz factor

$$
\gamma_{\max }=\frac{e E}{2 m_{\mathrm{e}} c}\left(\frac{B_{\|}}{\left\langle B_{\perp}\right\rangle} \frac{w a}{c v_{\mathrm{A}}}\right)^{1 / 2} \approx 5 \cdot 10^{6} .
$$

The resulting $\gamma_{\max }$ corresponds to an energy of about $3 \mathrm{TeV}$. This explains the observed optical synchrotron jet emission that appears to require $\gamma_{\max } \approx 10^{6}$. The maximum acceleration length $l_{\text {acc }}=c t_{\text {acc }} \approx 10^{16} \mathrm{~cm}$ is much less than either the length of the sheet $l_{\mathrm{cs}}$ or the synchrotron loss length. Thus, even for the highest electron energies, the strong DC electric field acceleration is a local acceleration mechanism that is not influenced by the synchrotron losses.

The interested reader can learn more about this work in Litvinenko (1999).

Acknowledgments. This work was supported by NSF grant ATM-9813933 and NASA grant NAG5-7792.

\section{References}

Eilek, J. A., \& Hughes, P. A. 1991, in Beams and Jets in Astrophysics, ed. P. A. Hughes (Cambridge: Cambridge Univ. Press), 428

Hill, T. W. 1975, J. Geophys. Res., 80, 4689

Litvinenko, Y. E. 1996, ApJ, 462, 997

Litvinenko, Y. E. 1999, A\&A, 349, 685 